\title{
Prevalência e fatores associados aos episódios de quedas em pessoas idosas no âmbito da Atenção Primária à Saúde
}

\section{Prevalence and factors associated with episodes of falls in elderly people in the scope of primary health care}

Luiz Henrique Batista Monteiro $^{1 *}$, Renata Di Pietro Carvalho ${ }^{1}$, Paulo Filipe de Mello ${ }^{1}$, Danielle Mandacaru Souza ${ }^{2}$, Rosana Passos Cambraia ${ }^{1}$, Renata Aline de Andrade ${ }^{1}$

\begin{abstract}
RESUMO
Objetivou estimar a prevalência e os fatores associados aos episódios de quedas em idosos que vivem em uma cidade polo do Vale do Jequitinhonha em Minas Gerais. Tratou-se de uma pesquisa transversal. A coleta de dados aconteceu entre os meses de janeiro 2018 a maio de 2019. Os dados foram analisados no programa Statistical Package for the Social Sciences. Participaram 313 idosos. A prevalência encontrada para quedas foi de $31,6 \%$. Os seguintes fatores associados foram identificados: idade maior que 80 anos, relato de problema crônico na coluna, autorrelato de diagnósticos de osteoporose, dependência para Atividades de Vida Diária e Dependência para Atividades Instrumentais de Vida Diária e polifarmácia. A prevalência encontrada se assemelha a outros achados e corrobora com a literatura nacional e internacional ao elucidar que os fatores associados são multicausais. É essencial a criação e implementação de estratégias que visem diminuir os episódios de quedas entre idosos, como por exemplo à educação e promoção à saúde.
\end{abstract}

Palavras-chave: Acidentes por quedas; Estudos transversais; Fatores de risco; Saúde do Idoso; Atenção integral ao idoso.

\section{ABSTRACT}

It aimed to estimate the prevalence and factors associated with episodes of falls in elderly people living in a pole city in Vale do Jequitinhonha in Minas Gerais. It was a cross-sectional survey. Data collection took place between January 2018 and May 2019. Data were analyzed using the Statistical Package for the Social Sciences program. 313 elderly people participated. The prevalence found for falls was 31.6\%. The following associated factors were identified: age over 80 years, report of chronic column problems, selfreport of diagnoses of osteoporosis, dependence for Activities of Daily Living and Dependence for Instrumental Activities of Daily Living and polypharmacy. The prevalence found is similar to other findings and corroborates the national and international literature by elucidating that the associated factors are multicausal. It is essential to create and implement strategies that aim to reduce episodes of falls among the elderly, such as education and health promotion.

Keywords: Accidental falls; Cross-sectional studies; Health services for the aged; Health of the Elderly; Risk factors.

\footnotetext{
${ }^{1}$ Universidade Federal dos Vales do Jequitinhonha e Mucuri

${ }^{2}$ Fundação Hospitalar São Vicente de Paulo

*E-mail: luizhbmonteiro@gmail.com
} 


\section{INTRODUÇÃO}

Episódios de quedas são comuns entre as pessoas idosas, porém, subnotificados (ANG et al., 2020). Além disso, a queda em idosos é uma importante síndrome geriátrica e constitui um tema preocupante para o cenário da saúde pública (FALSARELLA; GASPAROTTO; COIMBRA, 2014; LOHMAN et al., 2019). É um evento prevalente, que gera impacto negativo para a Qualidade de Vida (QV) (GALE; COOPER; SAYER, 2016; KHOW; VISVANATHAN; 2017; AMORIM et al., 2019; ANG et al., 2020 e maximiza as hospitalizações, nível de dependência e óbitos (GALE; COOPER; SAYER, 2016; KNOW; VISVANATHAN; 2017; AMORIM et al., 2019).

Infere-se que, em 2017, um a cada três idosos caíram pelo menos uma vez (VIEIRA et al., 2018). Corroborando com este estudo, aponta-se que no mesmo ano mais de seis milhões de idosos brasileiros sofreram quedas (PIMENTEL et al., 2018). No território brasileiro, em 2014, aconteceram 13.327 mortes provocadas por quedas, além de 1.118.048 hospitalizações por essa causa, sendo que destas, 26,1\% foram hospitalizações de pessoas idosas (RIBEIRO et al., 2016). Dados de um estudo realizado nos Estados Unidos da América, mostrou que em 2015 houve 28.486 mortes por quedas, e estimou-se que os custos médicos para estas mortes foram de 754 milhões de dólares (FLORENCE et al., 2018).

As quedas possuem impactos nos aspectos biopsicossociais e econômicos dos idosos e da sociedade (SMITH et al., 2017; AMORIM et al., 2021). No que diz respeito aos fatores associados as quedas, estes são multicausais (SMITH et al., 2017; KHOW; VISVANATHAN; 2017; PIMENTEL et al., 2018). Todavia, é imprescindível destacar que os fatores biológicos, comportamentais, ambientais e socioeconômicos são os principais motivadores de quedas na referida população (FALSARELLA; GASPAROTTO; COIMBRA, 2014). No campo da dimensão biológica, a redução da cognição e sensorial provocam as recidivas de quedas (AGMON et al., 2014).

Nesse sentido, é necessário a criação e implementação de intervenções factíveis com vista a minimizar as taxas de quedas e reconhecer os fatores de risco (KNOW; VISVANATHAN; 2017; CRUZ; LEITE, 2018; SHARIF et al., 2018). É primordial salientar que tais estratégias devem ser direcionadas majoritariamente aos grupos que se encontram suscetíveis para tal evento. Em outras palavras, idosos mais velhos, que têm baixa estratificação socioeconômica, que não possuem vínculo empregatício, além de idosos dependentes (VIEIRA et al., 2018; BARKER et al., 2019).

As medidas de prevenção a quedas incluem a atuação da equipe multiprofissional (KNOW; VISVANATHAN; 2017; AMORIM et al., 2021). Sobretudo na Atenção Primária à Saúde (APS), que pode contribuir para a minimização das quedas e de suas recorrências 
(NASCIMENTO; TAVARES, 2016; AMORIM et al., 2019; ANG et al., 2020). Compreendem ainda exemplos de atividades preventivas: o controle do uso de medicamentos, educação em saúde, o incentivo à prática de exercícios físicos e redução da incapacidade funcional (GASPAROTTO; FALSARELLA; COIMBRA, 2014). Espera-se que haja uma avaliação de quedas anualmente para idosos que apresentam predisposição para quedas (ANG et al., 2020).

Estimar a prevalência e os fatores associados aos episódios de quedas em idosos que vivem em uma cidade polo do Vale do Jequitinhonha em Minas Gerais.

\section{MÉTODOS}

Tratou-se de um estudo transversal, conduzido na área de abrangência de seis unidades de Estratégia Saúde da Família (eSF). Os dados foram coletados de janeiro de 2018 a maio de 2019. A amostra dos participantes deste estudo relacionou-se ao projeto de pesquisa "Sintomas depressivos e fatores associados em uma cidade polo do Vale do Jequitinhonha”. Para tal, realizou-se o cálculo amostral no OpenEpi (versão 3.01). Na época, haviam 1.791 idosos cadastrados em seis eSF, considerou-se a prevalência de 23,9\% de sintomas depressivos (BORGES et al., 2013), nível de significância de $95 \%(\alpha=0,05)$, erro de estimativa de $5 \%$, efeito de desenho de $1.0(10 \%)$, foram acrescidos $30 \%$ para perdas e controle de fatores de confusão. Sendo assim a amostra final foi composta por 313 idosos.

Foram incluídos nesta investigação idosos de ambos os sexos, com 60 anos ou mais, indivíduos que tinham capacidade cognitiva e auditiva para a compreensão das perguntas, idosos cadastrados na área de abrangência com Agente Comunitário de Saúde (ACS). Foram adotados como critérios de exclusão. Residir em Instituição de Longa Permanência para Idosos (ILPI) e idosos que estivessem sob efeito de alguma substância psicoativa ou hospitalizados.

Os participantes do estudo foram selecionados a partir da amostragem aleatória sistemática estratificada, fundamenta no cadastro da população no e-SUS Atenção Básica (e-SUS AB). Para o processo de aleatorização, considerou-se que a cada quatro domicílios, um idoso seria convidado. Nas residências com mais de um idoso habitando, o mais velho foi convidado a participar da pesquisa.

O questionário aplicado para a coleta dos dados sociodemográficos, situação de saúde e histórico de quedas foi advindo do EpiFloripa (BORGES et al., 2013; BORGES, 2014). Aplicouse ainda o index de Independência nas Atividades de Vida Diária (AVD), avalia a independência funcional do idoso para desempenhar seis funções (banho, vestir-se, ir ao banheiro, transferência, continência e alimentação). O instrumento classifica as pessoas como independentes ou dependentes paras as AVD (LINO et al., 2008). A Escala de Lawton foi aplicada para avaliação do desempenho funcional do indivíduo idoso no que se refere as atividades instrumentais que viabilizam ser independente para as atividades avaliadas. Neste instrumento, a pessoa idosa é classificada como independente ou dependente considerando nove funções. Para cada pergunta a 
primeira resposta diz respeito à independência, a segunda dependência parcial ou consegue com ajuda e a terceira dependência. A pontuação máxima é 27 pontos (LAWTON; BRODY, 1969).

Para avaliar a cognição dos idosos, aplicou-se o Mini Exame do Estado Mental (MEEM). As notas de corte dependem do nível educacional da pessoa que responde: analfabetos (20); 1 a 4 anos de escolaridade (25); 5 a 8 anos de escolaridade (26), 9 a 11 anos (28) $\geq 11$ anos (29) (BRUCKI et al., 2003). A Escala de Depressão Geriátrica (EDG-15) foi aplicada para avaliar a presença de sintomas depressivos. A pontuação até 5 indica ausência de sintomatologia depressiva e pontuação igual ou superior a seis aponte presença desses sintomas (ALMEIDA; ALMEIDA, 1999).

A variável desfecho desta investigação foi "queda no último ano". Foram consideradas variáveis independentes: sexo; idade; estado conjugal; idoso acamado, arranjo familiar; autopercepção da saúde; problema crônico na coluna, artrite ou reumatismo, osteoporose (BORGES et al., 2013; BORGES, 2014), dependência para ao menos uma AVD (LINO et al., 2008), dependência para ao menos uma AIVD (LAWTON; BRODY, 1969), Presença de sintomas depressivos (ALMEIDA; ALMEIDA, 1999), provável déficit cognitivo (BRUCKI et al., 2003) e polifarmácia (uso de cinco ou mais medicamentos) (LINJAKUMPU et al., 2002).

Os dados foram analisados no programa Statistical Package for the Social Sciences (SPSS), versão 23.0. Variáveis numéricas contínua foram descritas por média, intervalo de confiança de 95\% (IC 95\%) e desvio padrão (DP). Variáveis categóricas por meio de números absolutos, prevalências e IC 95\%. Realizou-se análise bivariada entre variáveis dependentes de independentes, com regressão logística, obtendo-se Odds Ratio (OR). As variáveis com p-value $<0,05$ foram consideradas associadas.

Todos os participantes da pesquisa assinaram o Termo de Consentimento Livre e Esclarecido (TCLE). A pesquisa que originou este trabalho foi aprovada pelo Comitê de Ética em Pesquisa (CEP) da Universidade Federal dos Vales do Jequitinhonha e Mucuri (UFVJM), sob protocolo $\mathrm{n}^{\circ} 2.268 .447 / 2017$.

\section{RESULTADOS}

Participaram deste estudo 313 idosos. A prevalência encontrada para quedas na presente investigação foi de 31,6\%. A média da idade entre os participantes foi de 71,9 (Desvio Padrão $(\mathrm{DP}) \pm 8,64)$ e a média para anos de estudo foi de 5,24 (DP $\pm 4,69)$ (dados não demonstrados na tabela). A tabela 1 apresenta os dados sociodemográficos da população do estudo.

Dos participantes, 61,7\% (IC95\%: 56,2-67,1) eram mulheres, 45,4\% (IC95\%: 39,9-51,1) tinham de 60 a 69 anos, 46,3\% (IC95\%: 40,6-51,8) informaram serem casados ou amasiados, 81,2\% (IC95\%: 76,7-85,6) relataram cor preta ou parda, 83,6\% (IC95\%: 79,2-87,2) sabiam ler e escrever, 88,8\% (IC95\%: 86,3-92,9) moravam com alguém, 93,0\% (IC95\%: 90,1-95,8 tinham renda, e 29,7\% (IC95\%: 24,5-34,8) 2 pessoas dependiam da renda 24,5-34,8. 
Tabela 1 - Caracterização sociodemográfica dos participantes. Cidade polo no Vale do Jequitinhonha, Minas Gerais, Brasil, (n=313), 2018-2019.

\begin{tabular}{|c|c|c|}
\hline VARIÁVEIS & $\mathbf{N}(\%)$ & IC $95 \%$ \\
\hline \multicolumn{3}{|l|}{ Sexo } \\
\hline Masculino & $120(38,3)$ & $32,9-43,8$ \\
\hline Feminino & $193(61,7)$ & $56,2-67,1$ \\
\hline \multicolumn{3}{|l|}{ Idade, em anos } \\
\hline $60-69$ & $142(45,4)$ & $39,9-51,1$ \\
\hline $70-79$ & $110(35,1)$ & $29,7-40,3$ \\
\hline 80 anos ou mais & $61(19,5)$ & $15,3-24$ \\
\hline \multicolumn{3}{|l|}{ Estado marital } \\
\hline Solteiro(a) & $30(9,6)$ & $6,4-12,8$ \\
\hline Divorciado(a)/Separado(a) & $26(8,3)$ & $5,4-11,5$ \\
\hline Viúvo & $112(35,8)$ & $30,4-41,5$ \\
\hline Casado(a)/Amasiado(a) & $145(46,3)$ & $40,6-51,8$ \\
\hline \multicolumn{3}{|l|}{ Cor da pele } \\
\hline Preta/Parda & $254(81,2)$ & $76,7-85,6$ \\
\hline Branca & $53(16,9)$ & $12,8-21,4$ \\
\hline Amarela & $6(1,9)$ & $0,6-3,5$ \\
\hline \multicolumn{3}{|l|}{ Sabe ler e escrever } \\
\hline Sim & $261(83,6)$ & $79,2-87,2$ \\
\hline Não & $52(16,6)$ & $12,8-20,8$ \\
\hline \multicolumn{3}{|l|}{ Mora } \\
\hline Só & $35(11,2)$ & $7,7-16,7$ \\
\hline Com alguém ${ }^{\mathrm{a}}$ & $278(88,8)$ & $86,3-92,9$ \\
\hline \multicolumn{3}{|l|}{ Tem renda ${ }^{b}$} \\
\hline Não & $22(7,0)$ & $4,2-9,9$ \\
\hline Sim & $291(93,0)$ & $90,1-95-8$ \\
\hline \multicolumn{3}{|l|}{ Dependentes da renda } \\
\hline Somente a pessoa idosa & $61(21,0)$ & $16,6-25,9$ \\
\hline 2 pessoas & $86(29,7)$ & $24,5-34,8$ \\
\hline 3 pessoas & $66(22,8)$ & $17,9-27,6$ \\
\hline 4 pessoas & $36(12,4)$ & $9,0-15,9$ \\
\hline 5 pessoas ou mais & $41(14,1)$ & $10,3-18,6$ \\
\hline
\end{tabular}

A tabela 2 apresenta as informações relacionados aos episódios de quedas. Entre os idosos que relataram episódio de queda no último ano, 57,6\% (IC95\%:47,5-67,7) caíram uma vez, 56,6\% (IC95:45,5-66,7) não procuraram atendimento médico, 37,4\% (IC95\%: 27,3-46,5) declararam 
que a queda ocorreu dentro do domicílio. 32,3\% (IC95\%23,2-41,4) comunicaram que estavam se locomovendo na ocasião da queda, 44,4\% (IC95: 34,3-54,5) escorregaram ou o calçado rompeu, 77,8\% (IC95\%:69,7-85,9) tiveram alguma consequência em razão da queda.

Tabela 2 - Informações dos episódios de quedas. Cidade polo no Vale do Jequitinhonha, Minas Gerais, Brasil, (n= 99), 2018-2019.

\begin{tabular}{|c|c|c|}
\hline VARIÁVEIS & $\mathbf{N}(\%)$ & IC95\% \\
\hline \multicolumn{3}{|l|}{ Quantidade de quedas no último ano } \\
\hline 1 & $57(57,6)$ & $47,5-67,7$ \\
\hline 2 & $22(22,2)$ & $14,1-30,3$ \\
\hline 3 ou mais & $20(20,2)$ & $13,1-28,3$ \\
\hline \multicolumn{3}{|l|}{ Procura por atendimento médico } \\
\hline Não & $56(56,6)$ & $45,5-66,7$ \\
\hline Algumas vezes & $31(31,3)$ & $22,2-41,4$ \\
\hline Todas as vezes & $12(12,1)$ & $6,1-19,2$ \\
\hline \multicolumn{3}{|l|}{ Local da queda } \\
\hline Dentro do domicílio & $37(37,4)$ & $27,3-46,5$ \\
\hline Quintal/jardim/pátio & $26(26,3)$ & $17,2-35,4$ \\
\hline Na rua/academia/oficina & $36(36,4)$ & $26,3-45,5$ \\
\hline \multicolumn{3}{|l|}{ Atividade que estava realizando } \\
\hline Tomando banho & $5(5,1)$ & $1,0-9,1$ \\
\hline Descendo/subindo escadas & $8(8,1)$ & $3,0-14,1$ \\
\hline Atividade doméstica & $24(24,2)$ & $15,2-32,3$ \\
\hline Locomovendo & $32(32,3)$ & $23,2-41,4$ \\
\hline Outros $^{\mathrm{a}}$ & $30(30,3)$ & $22,2-40,4$ \\
\hline \multicolumn{3}{|l|}{ Como a queda ocorreu } \\
\hline Pisou em falso & $10(10,1)$ & $5,1-16,2$ \\
\hline Desequilibrou-se & $11(11,1)$ & $5,1-18,2$ \\
\hline Tropeçou em algo & $14(14,1)$ & $7,1-21,2$ \\
\hline Sentiu-se tonto/fraco/desmaiou & $18(18,2)$ & $11,1-26,3$ \\
\hline Escorregou/calçado rompeu & $44(44,4)$ & $34,3-54,5$ \\
\hline Outros $^{b}$ & $2(2,0)$ & $0,0-5,1$ \\
\hline \multicolumn{3}{|l|}{ Tem medo de cair novamente ${ }^{b}$} \\
\hline Não & $11(12,0)$ & $6,6-18,7$ \\
\hline Sim & $80(87,9)$ & $81,3-93,4$ \\
\hline \multicolumn{3}{|l|}{ A queda gerou alguma consequência } \\
\hline Não & $22(22,2)$ & $14,1-30,3$ \\
\hline Sim & $77(77,8)$ & $69,7-85,9$ \\
\hline
\end{tabular}


O gráfico 1 expõe as consequências da queda. Dentre as possíveis consequências da queda, 47 idosos relataram escoriação, 44 idosos referiam hematomas, 22 disseram corte, 11 apresentaram fratura, 7 entorses e 6 outros (tonteira, desmaio, sangramento excessivo, tendinite e distensão do nervo.

Gráfico 1 - Consequências dos episódios de Quedas. Cidade polo do Vale do Jequitinhonha, Minas Gerais, Brasil, (n=99), 2018-2019.

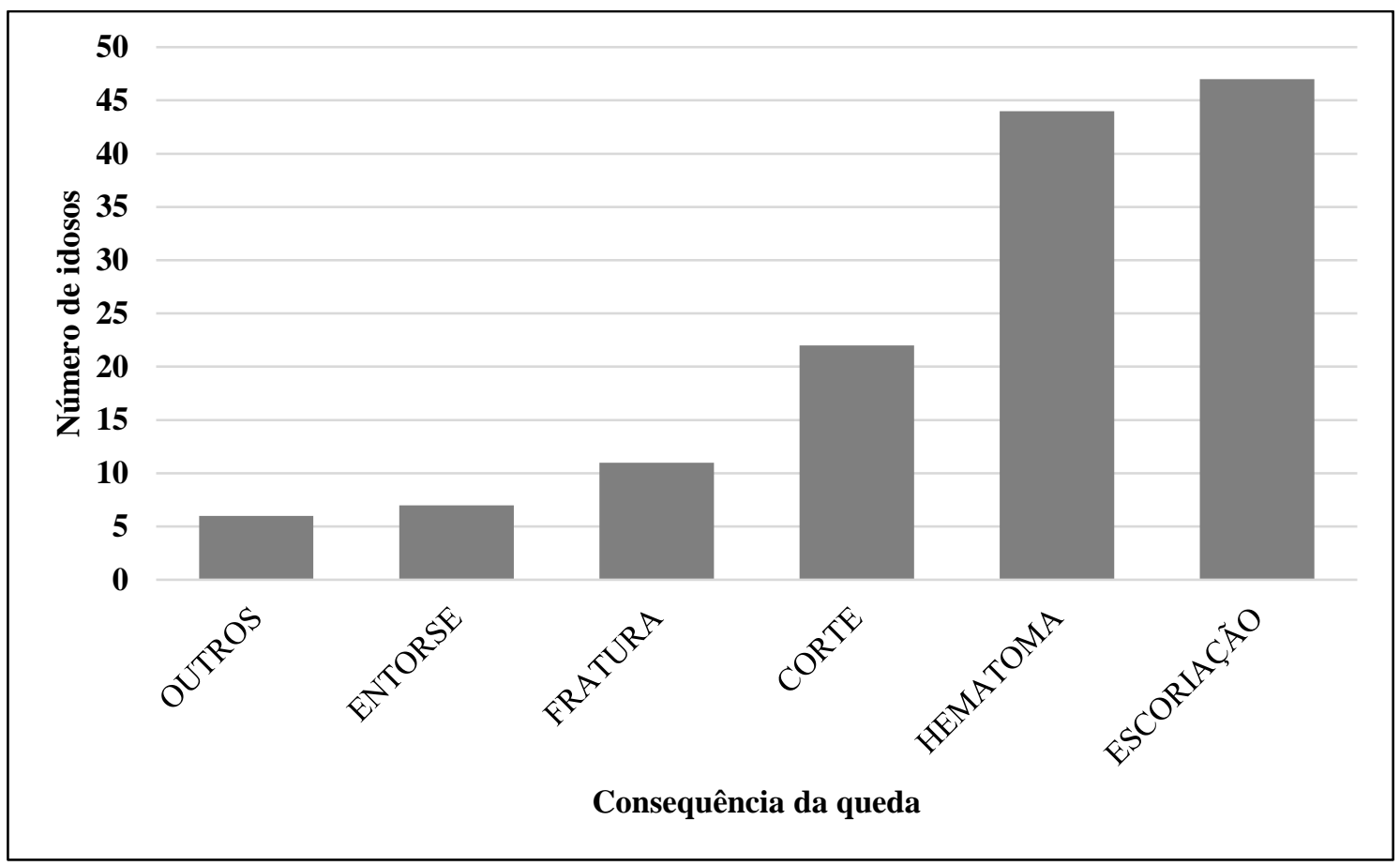

Fonte: Os autores.

A análise bivariada entre a variável dependente e independentes estão exibidas na tabela 3. Na análise bivariada as variáveis independentes associadas a variável preditora foram: faixa etária $\geq 80$ anos (OR 2,16 IC95\% 1,21-3,83), relato de diagnóstico de problema crônico na coluna (OR 1,76 IC95\% 1,08-2,84), autorrelato de diagnóstico de osteoporose (OR 1,20 IC95\% 0,672,13), dependência AVD (OR 4,53 IC95\% 1,11-18,53), dependência AIVD (OR 2,40 IC95\% 1,46-3,97) e polifarmácia (OR 1,89 IC95\% 1,17-3,07).

Tabela 3 - Análise bivariada e fatores associados aos episódios de quedas em idosos. Cidade polo no Vale do Jequitinhonha, Minas Gerais, Brasil, $(n=313), 2018-2019$.

\begin{tabular}{lcccc}
\hline & QUA* & \multicolumn{2}{c}{ OR bruta } \\
\hline Variável & n/total & $\%$ & (IC95\%) & Valor de p \\
\hline $\begin{array}{l}\text { Sexo } \\
\quad \text { Masculino }\end{array}$ & $37 / 120$ & 37,4 & 1,0
\end{tabular}




\begin{tabular}{|c|c|c|c|c|}
\hline & QUA* & & OR bruta & \\
\hline Variável & n/total & $\%$ & $(\mathrm{IC} 95 \%)$ & Valor de $p$ \\
\hline Feminino & $62 / 193$ & 62,6 & $1,06(0,65-1,73)$ & 0,81 \\
\hline \multicolumn{5}{|l|}{ Faixa etária } \\
\hline$<80$ anos & $71 / 252$ & 71,7 & 1,0 & \\
\hline$\geq 80$ anos & $28 / 61$ & 28,3 & $2,16(1,21-3,83)$ & 0,00 \\
\hline \multicolumn{5}{|l|}{ Estado conjugal } \\
\hline Vive com companheiro & $43 / 145$ & 43,4 & 1,0 & \\
\hline Vive sem companheiro & $56 / 168$ & 56,6 & $1,18(0,73-1,91)$ & 0,48 \\
\hline \multicolumn{5}{|l|}{ Escolaridade } \\
\hline$\leq 4$ anos & $72 / 204$ & 72,7 & 1,0 & \\
\hline$>4$ anos & $27 / 109$ & 27,3 & $0,60(0,35-1,01)$ & 0,05 \\
\hline \multicolumn{5}{|l|}{ Arranjo familiar } \\
\hline Mora acompanhado & $89 / 278$ & 89,9 & 1,0 & \\
\hline Mora sozinho & $10 / 35$ & 10,1 & $0,84(0,38-1,84)$ & 0,68 \\
\hline \multicolumn{5}{|l|}{ Acamado } \\
\hline Não & $96 / 207$ & 96,0 & 1,0 & \\
\hline Sim & $3 / 6$ & 3,0 & $2,19(0,43-11,08)$ & 0,32 \\
\hline \multicolumn{5}{|c|}{ Avaliação da situação de saúde } \\
\hline Positiva & $45 / 165$ & 45,5 & 1,0 & \\
\hline Negativa & $54 / 148$ & 54,5 & $1,53(0,94-2,47)$ & 0,08 \\
\hline \multicolumn{5}{|c|}{$\begin{array}{l}\text { Relato de diagnóstico de problema crônico } \\
\text { na coluna }\end{array}$} \\
\hline Não & $43 / 166$ & 43,4 & 1,0 & \\
\hline Sim & $56 / 147$ & 56,6 & $1,76(1,08-2,84)$ & 0,02 \\
\hline \multicolumn{5}{|l|}{ Artrite ou reumatismo } \\
\hline Não & $52 / 185$ & 52,5 & 1,0 & \\
\hline Sim & $47 / 123$ & 47,5 & $1,48(0,91-2,40)$ & 0,10 \\
\hline \multicolumn{5}{|c|}{ Autorrelato de diagnóstico de Osteoporose } \\
\hline Não & $74 / 258$ & 74,7 & 1,0 & \\
\hline Sim & $25 / 55$ & 23,2 & $1,20(0,67-2,13)$ & 0,01 \\
\hline \multicolumn{5}{|l|}{ Dependência AVDa } \\
\hline Não & $93 / 304$ & 93,9 & 1,0 & \\
\hline Sim & $6 / 9$ & 6,1 & $4,53(1,11-18,53)$ & 0,02 \\
\hline \multicolumn{5}{|l|}{ Dependência AIVDa } \\
\hline Não & $54 / 212$ & 54,5 & 1,0 & \\
\hline Sim & $45 / 100$ & 45,5 & $2,40(1,46-3,97)$ & 0,00 \\
\hline \multicolumn{5}{|c|}{ Presença de sintomas depressivos } \\
\hline Não & $73 / 246$ & 73,7 & 1,0 & \\
\hline Sim & $26 / 67$ & 26,3 & $1,50(0,85-2,62)$ & 2,03 \\
\hline \multicolumn{5}{|l|}{ Provável déficit cognitivo } \\
\hline Não & $34 / 103$ & 30,3 & 1,0 & \\
\hline Sim & $64 / 210$ & 69,7 & $1,19(0,71-1,99)$ & 0,86 \\
\hline \multicolumn{5}{|l|}{ Polifarmácia } \\
\hline Não & $44 / 173$ & 44,4 & 1,0 & \\
\hline Sim & $55 / 140$ & 55,6 & $1,89(1,17-3,07)$ & 0,00 \\
\hline
\end{tabular}

*QUA: Queda no último ano, a: dependência para uma ou mais atividades.

\section{DISCUSSÃO}

Este estudo inovou ao apresentar os fatores associados aos episódios de quedas em idosos residentes no Vale do Jequitinhonha. Na análise, foram identificados os seguintes fatores de risco: 
idade maior ou igual a 80 anos, polifarmácia, relato de diagnóstico de problema crônico na coluna, autorrelato de diagnóstico de Osteoporose, dependência AVD e dependência AIVD.

A prevalência de quedas identificada nesta investigação foi de $31,6 \%$, achado que se aproxima a outros resultados no Brasil, que apontam que a prevalência varia de $27,1 \%$ a $37,5 \%$ (AMORIM et al., 2019; CRUZ; LEITE, 2018; PIMENTEL et al., 2018; NASCIMENTO; TAVARES, 2016; SOARES et al., 2014). No entanto, a prevalência encontrada neste estudo é menor em comparação aos achados de Natal e Goiânia, que identificaram a prevalência de 53,6\%, 60,5\%, respectivamente (VERA et al., 2015; SANTOS et al., 2015). Estudo conduzido em Dubai, nos Emirados Árabes, identificou que 50,8\% dos participantes possuíam histórico de quedas (SHARIF et al., 2018). Já na Índia, a prevalência oscilou de $26 \%$ a $37 \%$ a depender da região (JOSEPH; KUMAR; BAGAVANDAS, 2019).

Quanto ao número de quedas, 57,6\% dos idosos anunciaram um episódio de queda. Resultado semelhante ao encontrado em outro estudo (VIEIRA et al., 2018). Um dado que merece atenção, diz respeito que $42,4 \%$, tiveram duas ou mais queda, corroborando aos achados de Vieira et al. (2018). Esses achados demonstram quão habitual são as recidivas de quedas na população idosa. Em relação ao local da queda, 37,4\% afirmaram que a queda aconteceu na habitação e $36,4 \%$ na rua/academia/oficina. Estudo de Silva-Fhon e colaboradores (2019) identificaram que 47,7\% das quedas aconteceram dentro de casa, especificamente na sala e nos quartos. Na pesquisa realizada em Pelotas-RS, 56,4\% dos idosos informaram que a queda aconteceu na morada e 46,6\% na rua (VIEIRA et al., 2018). Já em Hanói, Vietnã, 69,6\% das quedas deram-se no lar (HA et al., 2021).

Adicionalmente, 32,3\% informaram que estavam se locomovendo no momento da queda, desfecho similar a outro resultado (OLIVEIRA et al., 2014). O estudo evidenciou que 44,4\% dos idosos caíram por terem escorregado ou o calçado rompeu. Pesquisa semelhante verificou que $51,6 \%$ das quedas relatadas pelos idosos, ocorreram pelo chão estar escorregadio (HA et al., 2021).

Nesta investigação, entre os participantes que relataram quedas, 77,8\% referiram alguma consequência em virtude do ato de cair. Nada obstante, 56,6\% não procuraram atendimento médico. No estudo realizado na capital do Peru, no que tange as consequências, identificou-se a dificuldade para deambular, receio de cair novamente e mudança no domicílio, dos quais $9,1 \%$ necessitaram de hospitalização (SILVA-FHON et al., 2019).

O presente estudo revelou que $87,9 \%$ dos idosos relataram medo de cair novamente. É esperado que este público tenha receio de cair novamente, principalmente, após graves consequências, que ocasionem restrição das atividades diárias e instrumentais e reduza a mobilidade (SANTOS et al., 2015). Em uma metanálise, realizada com 3.112 idosos, observouse que o risco para queda oscilou de 12 a 15 vezes na população idosa que verbalizou medo de cair (PENA et al., 2019). O medo de cair possui relação direta a quedas recorrentes (SOUZA et 
al., 2019). As quedas, além do medo de cair novamente, podem suscitar síndrome de ansiedade pós-quedas, depressão e diminuição de atividades diárias, gerando assim, impacto negativo no bem-estar (ANG et al., 2020).

No que se refere as consequências, as mais prevalentes nesse estudo foram: escoriação, hematoma e corte, respectivamente. Numa pesquisa realizada no Sul do Brasil, identificou-se que $12,0 \%$ das pessoas idosas participantes fraturaram algum osso. Sendo que 4,9\% relataram fratura em membros inferiores e 3,9\% nos membros superiores (VIEIRA et al., 2018). As mulheres tendem a ferimentos mais graves (HA et al., 2021). Em Lima, 59,1\% das pessoas idosas foram identificadas com algum ferimento (SILVA-FHON et al., 2019).

Revela-se que nesta investigação, a idade maior que 80 anos, foi um fator associado para o episódio de quedas. Logo, este resultado ratifica com outros estudos (GALE; COOPER; SAYER, 2016; SOARES et al., 2014; NASCIMENTO; TAVARES, 2016; CRUZ; LEITE, 2018; OLIVEIRA, BAIXINHO; HENRIQUES, 2019; SILVA-FHON et al., 2019). Possivelmente, essa associação ocorra em razão da diminuição da massa muscular, dado que, indivíduos mais velhos tendem a andar mais devagar (GALE; COOPER; SAYER, 2016). Além disto, relaciona-se ao declínio físico e funcional que esses indivíduos apresentam (SOARES et al., 2014).

O autorrelato de diagnóstico de osteoporose associou-se aos episódios de quedas. São agentes causais do envelhecimento musculoesquelético, os fatores relacionados à idade, inflamações, mudança da massa corpórea e desequilíbrios hormonais. A osteoporose está associada a síndrome de fragilidade, que predispõe aos indivíduos incapacidade funcional, doenças cardiovasculares, cânceres, mortalidade e quedas (GRECO; PIETSCHMANN; MIGLIACCIO, 2019).

Na presente investigação, ter problema crônico de coluna constituiu-se fator associado a quedas. Outros estudos similares apontam esta associação (JAGT-WILLEMS et al., 2015; NASCIMENTO; TAVARES, 2016; SMITH et al., 2017). Em uma coorte identificou-se que $40 \%$ dos pacientes com hipercifose tiveram uma ou mais fraturas vertebrais (JAGT-WILLEMS et al., 2015).

Observou-se neste estudo que as dependências para AIVD e AVD foram associadas a quedas. Resultado equivalente a outros estudos (NASCIMENTO; TAVARES, 2016; VIEIRA et al., 2018). Pesquisas apontam que idosos, após experiência de queda, normalmente tendem a diminuir AVD e AIVD, o que impacta negativamente na QV e bem-estar desses indivíduos, que acabam recorrendo a um suporte familiar maior. Apesar disso, a pouca limitação funcional nas AVD e a participação social são aspectos positivos para essa população. Mulheres ativas tendem a apresentar melhores escores de QV, com maior participação social, maior renda e não sofrem quedas (SILVA et al., 2012; CAMPOS; FERREIRA; VARGAS, 2015).

A polifarmácia associou-se às quedas. Outros estudos apontaram esta mesma associação (NASCIMENTO; TAVARES, 2016; LAWSON et al., 2018; GEORGE; VERGHESE, 2018). 
Sabe-se que o envelhecer pode estar associado a comorbidades e condições que favorecem ao uso concomitante de múltiplos medicamentos (SECOLI, 2010; CAMERON et al., 2018; LAWSON et al., 2018). Em relação as classes dos medicamentos, o uso e consequentemente interação entre benzodiazepínicos, anticolinérgicos, beta-bloqueadores e neurolépticos, favorece a quedas em idosos (SECOLI, 2010).

Trabalhos demonstraram que tomar quatro ou mais medicamentos se associa ao aumento da incidência de quedas e dessa maneira, amplia a reincidência de quedas e suas consequências (ZIA; KAMARUZZAMAN; TAN, 2015). Em virtude das quedas, é indispensável que os prescritores revisem periodicamente a farmacoterapia (LAWSON et al., 2018). Isto posto, o uso de medicações corretas e revisão periódica das medicações são substanciais para limitar o impacto da polifarmácia nas quedas (ZIA; KAMARUZZAMAN; TAN, 2015).

O estudo apresenta algumas limitações. Primeiro, o método empregado, uma vez que estudo transversal é incipiente para desenhar conclusões sobre a causalidade. Segundo, as amostras foram recrutadas de uma única cidade. À vista disso, os resultados podem não representar todos os idosos do Vale do Jequitinhonha. Terceiro, elenca-se o viés de memória, visto que a variável desfecho relacionou-se ao último ano.

\section{CONCLUSÃO}

Esta investigação identificou a prevalência significativa para quedas entre os participantes, resultado que se aproxima a outros estudos. Identificou que idade maior que 80 anos, polifarmácia, problema crônico na coluna, osteoporose, dependência para AVD e dependência para AIVD, foram fatores associados. Esse resultado reforça que as quedas são constantes na população idosa e que não há um único fator responsável.

Nesse sentido, é indispensável a criação e implementação de estratégias que visem diminuir os episódios de quedas entre idosos, como por exemplo educação em saúde e promoção à saúde. Além disto, é substancial que os profissionais de saúde, sobretudo os atuantes na APS, estejam habilitados para atender vítimas de quedas, prevenir tais episódios e promover a saúde dos idosos.

\section{REFERÊNCIAS}

AGMON, M.; BELZA, B.; NGUYEN, H. Q.; LOGSDON, R. G.; KELLY, V. E. A systematic review of interventions conducted in clinical or community settings to improve dual-task postural control in older adults. Clinical Interventions in Aging, Auckland, v. 9, p. 477-492, 2014.

ALMEIDA, O. P.; ALMEIDA, A. S. Confiabilidade da versão brasileira da Escala de Depressão em Geriatria (GDS) versão reduzida. Arquivo de Neuropsiquiatria, v. 57, n. 2, p. 421-426, 1999. 
AMORIM, J. S. C.; TORRES, K. C. L.; TEIXEIRA-CARVALHO, A.; MARTINSPINTO, O. A.; LIMA-COSTA, M. F.; PEIXOTO, S. V. Marcadores inflamatórios e ocorrência de quedas: coorte de idosos de Bambuí. Revista de Saúde Pública, São Paulo, v. 53, n. 35, p. 1-11, 2019.

AMORIM, J. S. C.; SOUZA, M. A. N.; MAMBRINI, J. V. M.; LIMA-COSTA, M. F.; PEIXOTO, S. V. Prevalência de queda grave e fatores associados em idosos brasileiros: resultados da Pesquisa Nacional de Saúde, 2013. Ciência \& Saúde Coletiva, v. 26, n. 1, p. 185-196, 2021.

ANG, G. C.; LOW, S. L.; HOW, C. H. Approach to falls among the elderly in the Community. Singapore Med J, v. 61, n. 3, p. 116-121, 2020.

BARKER, A.; CAMERON, P.; FLICKER, L.; ARENDTS, G.; BRAND, C.; ETHERTON-BEER, C. et al. Evaluation of respond, a patient-centred program to prevent falls in older people presenting to the emergency department with a fall: a randomised controlled trial. PLoS Medicine, San Francisco, v. 16, n. 5, e1002807, p. 118, 2019.

BORGES, L. J. Sintomas depressivos e atividade física em idosos: estudo longitudinal. 2014. 183f. Tese (Doutorado em Educação Física) - Universidade Federal de Santa Catarina, Florianópolis, 2014.

BORGES, L. J.; BENEDETTI, T. R. B.; XAVIER, A. J.; D’ORSI, E. Fatores associados aos sintomas depressivos em idosos: EpiFloripa. Revista de Saúde Pública, São Paulo, v. 47, n. 4, p. 701-710, 2013.

BRUCKI, S. M. D.; NITRINI, R.; CARAMELLI, P.; BERTOLUCCI, P. H.; OKAMOTO, I. H. Sugestões para o uso do Mini-Exame do Estado Mental no Brasil. Arquivo de Neuropsiquiatria, São Paulo, v. 61, n. 3b, p. 777-781, 2003.

CAMERON, E. J.; BOWLES, S. K.; MARSHALL, E. G.; ANDREW, M. K. Falls and long-term care: a report from the care by design observational cohort study. BMC family practice, Londres, v. 19, n. 73, p. 1-7, 2018.

CAMPOS, A. C. V.; FERREIRA, E. F.; VARGAS, A. M. D. Determinantes do envelhecimento ativo segundo a qualidade de vida e gênero. Ciência \& Saúde Coletiva, Rio de Janeiro, v. 2, n. 7, p. 2221-2237, 2015.

CRUZ, D. T.; LEITE, I. C. G. Quedas e fatores associados em idosos residentes na comunidade. Revista Brasileira de Geriatria e Gerontologia, Rio de Janeiro, v. 21, n. 5, p. 551-561, 2018.

FALSARELLA, G. R.; GASPAROTTO, L. P. R.; COIMBRA, A. M. V. Quedas: conceitos, frequências e aplicações à assistência ao idoso. Revisão da literatura. Revista Brasileira de Geriatria e Gerontologia, Rio de Janeiro, v. 17, n. 4, p. 897-910, 2014.

FERNANDES, M. G. M.; BARBOSA, K. T. F.; OLIVEIRS, F. M. R. L.; RODRIGUES, M. M. D. SANTOS, K. F. O. Risco de quedas evidenciado por idosos atendidos num ambulatório de geriatria. Rev. Eletr. Enf. [Internet], v. 16, n. 2, p. $297-$ 303, abr/jun 2014. 
FLORENCE, C. S.; BERGEN, G.; ATHERLY, A.; BURNS, E.; STEVENS, J.; DRAKE, C. The medical costs of fatal falls and fall injuries among older adults. Am Geriatr Soc, v. 66, n. 4, p. 693-698, abr. 2018.

GALE, C. R.; COOPER, C.; SAYER, A. A. Prevalence and risk factors for falls in older men and women: The English Longitudinal Study of Ageing. Age Ageing, Londres, v. 45, n. 6, p. 789-794, jul. 2016.

GASPAROTTO, L. P. R.; FALSARELLA, G. R.; COIMBRA, A. M. V. As quedas no cenário da velhice: conceitos básicos e atualidades da pesquisa em saúde. Revista Brasileira de Geriatria e Gerontologia, Rio de Janeiro, v. 17, n. 1, p. 201-209, 2014.

GEORGE, C. J.; VERGHENSE, J. Polypharmacy and Gait Performance in Community Dwelling Older Adults. Am Geriatr Soc, v. 65, n. 9, p. 2082-2087, sep. 2017.

GRECO, E. A.; PIETSCHMANN, P.; MIGLIACCIO, S. Osteoporosis and Sarcopenia Increase Frailty Syndrome in the Elderly. Frontiers in endocrinology, Suíça, v. 10, p. 1-10, abr. 2019.

HA, V. T.; NGUYEN, T. N.; NGUYEN, T. X.; NGUYEN, H. T. T.; NGUYEN, T. T. H.; NGUYEN, A. T. et al. . Prevalence and factors associated with falls among older outpatients. Int. J. Environ. Res. Public Health, v. 18, 4041, 2021.

HOPEWELL, S.; COPSEY, B.; NICOLSON, P.; ADEDIRE, B.; BONIFACE, G.; LAMB, S. Multifactorial interventions for preventing falls in older people living in the community: a systematic review and meta-analysis of 41 trials and almost 20000 participants. Br J Sports Med, v. 54, p. 1340-1350, 2020.

JAGT-WILLEMS, H. C.; GROOT, M. H.; CAMPEN, J.; LAMOTH, C. J. C.; LEMS, W. F. Associations between vertebral fractures, increased thoracic kyphosis, a flexed posture and falls in older adults: a prospective cohort study. Biomed Center Geriatrics, Londres, v. 15, n. 34, p. 1-6, 2015.

JOSEPH, A.; KUMAR, D.; BAGAVANDAS, M. A review of epidemiology of fall among elderly in India. J Community Med, v. 44, p.166-168, 2019.

KHOW, K. S. F.; VISVANATHAN, R. Falls in the aging population. Clinics in Geriatric Medicine, United States, v. 33, n. 3, p. 357-68, 2017.

LAWSON, K.; VINLUAN, C. M.; OGANESYAN, A.; GONZALEZ, E. C.; LOYA, A.; STRATE, J. J. A retrospective analysis of prescription medications as it correlates to falls for older adults. Pharmacy Practice, Granada, v. 16, n. 4, p. 1-6, oct./dec. 2018.

LAWTON, M. P.; BRODY, E. M. Assessment of older people: self-maintaining and instrumental activities of daily living. The Gerontologist, United States, v. 9, n. 3, p. 179-186, 1969.

LINJAKUMPU, T. HARTIKAINEN, S.; KLAUKKA, T.; VEIJOLA. J.; KIVELA, S. L.; ISOAHO, R. Use of medications and polypharmacy are increasing among the elderly. Journal of Clinical Epidemiology, United States, v. 55, n. 8, p. 809-817, ago. 2002. 
LINO, V. T. S.; PEREIRA, R. M.; CAMACHO, L. A. B.; RIBEIRO FILHO, S. T.; BUSKSMAN, S. Adaptação transcultural da Escala de Independência em Atividades da Vida Diária (Escala de Katz). Cadernos de Saúde Pública, Rio de Janeiro, v. 24, n. 1, p. 103-112, jan. 2008.

LOHMAN, M. C.; SONNEGA, A. J.; NICKLETT, E. J.; ESTENSON, L.; LEGGETT, A. N. Comparing Estimates of Fall-Related Mortality Incidence Among Older Adults in the United States. J Gerontol A Biol Sci Med Sci., v. 74, p. 1468-1474, 2019.

NASCIMENTO, J. S.; TAVARES, D. M. S. Prevalência e fatores associados a quedas em idosos. Texto Contexto Enfermagem, Florianópolis, v. 25, n. 2, e0360015, p. 1-9, 2016.

OLIVEIRA, A. S.; TREVIZAN, P. F.; BESTETTI, M. L. T.; MELO, R. C. Fatores ambientais e risco de quedas em idosos: revisão sistemática. Rev. Bras. Geriatr. Gerontol., Rio de Janeiro, v. 17, n. 3, p. 637-645, 2014.

OLIVEIRA, T.; BAIXINHO, C. L.; HENRIQUES, M. A. Risco multidimensional de queda em idosos Revista Brasileira em Promoção da Saúde, Fortaleza, v. 31, n. 2, p. 1-9, abr./jun., 2018.

PENA, S. P.; GUIMARAES, H. C. Q. C. P.; LOPES, J. L.; GUANDALINI, L. S.; TAMINATO, M.; BARBOSA, D. A. et al. Medo de cair e o risco de queda: revisão sistemática e metanálise. Acta Paulista de Enfermagem, São Paulo, v. 32, n. 4, p. 456463, 2019.

PIMENTEL, W. R. T.; PAGOTTO, V.; STOPA, S. R.; HOFFMANN, M. C. C. L.; ANDRADE, F. B.; SOUZA JUNIOR, P. R. B. et al. L. Quedas entre idosos brasileiros residentes em áreas urbanas: ELSI-Brasil. Revista de Saúde Pública, São Paulo, v. 52, Supl. 2, p. 1-12, 2018.

REIS, K. M. C.; JESUS, C. A. C. Relação da polifarmácia e polipatologia com a queda de idosos institucionalizados. Texto Contexto Enferm, v. 26, n. 2, e3040015, 2017.

RIBEIRO, A. R.; SOUZA, E. R.; SOUSA, C. A. M.; FREITAS, M. G. Quedas acidentais nos atendimentos de urgência e emergência: resultados do VIVA Inquérito de 2014. Ciência \& Saúde Coletiva, Rio de Janeiro, v. 21, n. 12, p. 3719-3727, 2016.

SANTOS, R. K. M.; MACIEL, A. C. C.; BRITTO, H. M. J. S.; LIMA, J. C. C.; SOUZA, T. O. Prevalência e fatores associados ao risco de quedas em idosos adscritos a uma Unidade Básica de Saúde do município de Natal, RN, Brasil. Ciência \& Saúde Coletiva, Rio de Janeiro, v. 20, n. 12, p. 3753-3762, 2015.

SECOLI, S. R. Polifarmácia: interações e reações polifarmácia: interações e reações adversas no uso de medicamentos por idosos. Revista Brasileira de Enfermagem, Brasília, v. 63, n. 1, p. 136-140, jan./fev. 2010.

SHARIF, S. I.; AL-HARBI, A. B.; AL-SHIHABI, A. M.; AL-DAOUR, D. S.; SHARIF, R. S. Falls in the elderly: assessment of prevalence and risk factors. Pharmacy Practice, Granada, v. 16, n. 3, p. 1-7, jul./set. 2018. 
SILVA, A.; FALEIROS, H. H. F.; SHIMIZU, W. A. L.; NOGUEIRA, L. M.; NHAN, L. L.; SILVA, B. M. F. et al. Prevalência de quedas e de fatores associados em idosos segundo etnia. Ciência \& Saúde Coletiva, Rio de Janeiro, v. 17, n. 8, p. 2181-2190, 2012.

SILVA-FHON, J. R.; PARTIENAZI-RODRIGUES, R.; MIAYAMURA, K,. FUENTES-NEIRA, W. Causas y factores asociados a las caídas del adulto mayor. Enfermería Universitaria, México, v. 16, n. 1, p. 31-40, jan./mar., 2019.

SMITH, A. A.; SILVA, A. O.; RODRIGUES, R. A. P.; MOREIRA, M. A. S. P.; NOGUEIRA, J. A.; TURA, L. F. R. Avaliação do risco de quedas em idosos residentes em domicílio. Revista Latino-Americana de Enfermagem, Ribeirão Preto, v. 25, e2754, p. 1-9, 2017.

SOARES, W. J. S.; MORAES, S. A.; FERRIOLI, E.; PERRACINI, M. R. Fatores associados a quedas e quedas recorrentes em idosos: estudo de base populacional. Revista Brasileira de Geriatria e Gerontologia, Rio de Janeiro, v. 17, n. 1, p. 49-60, 2014.

SOUZA, A. Q.; PEGORARI, M. S.; NASCIMENTO, J. S.; OLIVEIRA, P. B.; TAVARES. Incidência e fatores preditivos de quedas em idosos na comunidade: um estudo longitudinal. Ciência \& Saúde Coletiva, p. 24, n. 9, p. 3507-3516, 2019.

VERA, I.; LUCCHESE, R.; NAKATANI, A. Y. K.; SADOYAMA, G. BACHION, M. M.; VILA, V. S. C Fatores associados à disfuncionalidade familiar em idosos não institucionalizados. Texto \& Contexto Enfermagem, Florianópolis, v. 24, n. 2, p. 494504, abr./jun. 2015.

VIEIRA, L. S.; GOMES, A. P.; BIERHALS, I. O.; FARIAS-ANTUNEZ, S.; RIBEIRO, C. G.; MIRANDA, V. I. LUTZL. et al. Quedas em idosos no Sul do Brasil: prevalência e determinantes. Revista de Saúde Pública, São Paulo, v. 52, n. 22, p. 1-13, 2018.

ZIA, A.; KAMARUZZAMAN, S. B.; TAN, M. P. Polypharmacy and falls in older people: Balancing evidence-based medicine against falls risk. Postgraduate Medicine, v. 127. n. 3, p. 330-337, 2015.

Recebido em: 15/01/2022

Aprovado em: 05/02/2022

Publicado em: 10/02/2022 